\title{
Small Bowel Adenocarcinoma Presenting with Refractory Iron Deficiency Anemia - Case Report and Review of Literature
}

\author{
Nishant Poddar ${ }^{\mathrm{a}}$ Shahzad Raza ${ }^{\mathrm{a}}$ Bhawna Sharma ${ }^{\mathrm{a}}$ \\ Ming Liu ${ }^{b}$ Arash Goharic Madhumati Kalavar ${ }^{a}$ \\ Divisions of a Hematology and Oncology, ${ }^{b}$ Pathology, and ${ }^{\mathrm{c}}$ Radiology, Brookdale \\ University Hospital and Medical Center, Brooklyn, N.Y., USA
}

\section{Key Words}

Small bowel adenocarcinoma $\cdot$ FOLFOX · Iron deficiency anemia

\begin{abstract}
Cancers of the small bowel are relatively rare and account for approximately $1-2 \%$ of all gastrointestinal neoplasms. The most common histologic subtype - adenocarcinoma constitutes $40 \%$ of all cases. These cancers generally present with vague abdominal discomfort and are often diagnosed at a late stage and carry a poor prognosis. The treatment of choice of early-stage small bowel adenocarcinoma is surgical resection. No standard treatment protocol has been defined for unresectable or metastatic disease. Here, we report a case of a 56-year-old woman who presented with unexplained iron deficiency anemia. Extensive initial studies with serial CT scans of the abdomen, esophagogastroduodenoscopy, small bowel capsule endoscopy and colonoscopy were noncontributory. She was later found to have a metastatic small bowel adenocarcinoma and treated with palliative chemotherapy. She achieved a modest response to the treatment. Interestingly, in our case, the sole presentation was unexplained iron deficiency anemia. Physician's awareness regarding the possibility of small bowel cancer especially in the setting of iron deficiency and its workup has been emphasized. This enhances the chance of early detection and hence better survival.
\end{abstract}

\section{Background}

Malignant neoplasms of the small bowel are exceedingly rare and account for only $2 \%$ of all gastrointestinal cancers [1]. The most commonly occurring histological subtypes are adenocarcinomas (40\%), malignant carcinoid tumors (36\%), lymphomas and sarcomas 
[1]. The peak incidence of small bowel tumor is in the fifth and sixth decade of life with a higher prevalence rates in black population than in whites ( $10.6 \mathrm{vs.} 5.6$ per million populations) [3]. At present, there are no good screening methods for small bowel adenocarcinoma (SBA). Initial symptoms of SBA are vague, nonspecific, and hence appropriate tests are not ordered. The current methodologies for examining the small bowel have proved inadequate. In spite of recent advances in radiographic imaging and endoscopy techniques, early detection of neoplasms of the small intestine is still low [4]. Therefore, the diagnosis of small bowel cancers is often delayed, contributing to the poor prognosis.

Epidemiologically, SBAs are more common at the proximal end. Approximately $50 \%$ of the cases arise in the duodenum, 30\% in the jejunum, and 20\% in the ileum. Genetic analyses suggest $K$-ras mutation and $p 53$ overexpression are common in SBA as they are in colorectal carcinoma $[5,6]$.

To our knowledge, little is known about the clinical characteristics, treatment modalities and prognosis of patients with SBA. This is mainly due to the rarity of this cancer. Maglinte et al. [7] have shown that there is a 8.2-month delay in the diagnosis of SBA, due to its vague symptoms like nonspecific abdominal discomfort. This is attributed to the failure of the physician to order the appropriate tests. Therefore, it is very important to identify the cause of unexplained iron deficiency anemia in middle-aged patients as it could possibly be a harbinger for SBA. Here, we report a case of SBA presenting with unexplained iron deficiency anemia for a prolonged period, with classical noncontributory radiography, gastroduodenoscopy, small bowel capsule endoscopy and colonoscopy.

\section{Case Report}

A 56-year-old woman with a one-year history of unexplained iron deficiency anemia despite extensive gastrointestinal workup was admitted with dizziness and shortness of breath. She was found to have severe anemia (hemoglobin $5 \mathrm{~g} / \mathrm{dl}$ ) in the emergency room. Her past medical history includes chronic smoking, hemoglobin $\mathrm{C}$ trait and hypertension which was diet controlled. She denied any recent weight loss, constipation, melena or hematemesis. One year prior to her admission, her upper gastrointestinal endoscopy and colonoscopy as a workup for iron deficiency anemia were normal. She later had a video-assisted capsule endoscopy which was also unremarkable. Interestingly, CT scan of abdomen and pelvis with oral and intravenous contrast 4 months and 1 year prior to her admission were normal as well.

On physical examination, she was afebrile with stable vital signs. She appeared cachectic with severe pallor of the conjunctiva. Physical examination was otherwise unremarkable. Her abdomen was soft without any rebound tenderness and did not reveal any organomegaly. Laboratory tests showed (reference range in parentheses) severe microcytic hypochromic anemia with hemoglobin of $5.0 \mathrm{~g} / \mathrm{dl}$ $(11.7-16.1 \mathrm{~g} / \mathrm{dl})$, hematocrit of $15.9 \%$ (37-51\%), mean corpuscular volume of $66.5 \mathrm{fl}(80-101 \mathrm{fl})$, platelet count of $546 \times 10^{9} / \mathrm{l}\left(130-450 \times 10^{9} / \mathrm{l}\right)$, serum ferritin of $6.28 \mathrm{ng} / \mathrm{ml}(11-264 \mathrm{ng} / \mathrm{ml})$, transferrin of 237 $\mathrm{mg} / \mathrm{dl}(250-380 \mathrm{mg} / \mathrm{dl})$, total iron binding capacity of $297 \mu \mathrm{g} / \mathrm{dl}(240-450 \mu \mathrm{g} / \mathrm{dl})$, vitamin $\mathrm{B}_{12}$ of 540 $\mathrm{pg} / \mathrm{ml}(239-931 \mathrm{pg} / \mathrm{ml})$, reticulocyte count of $2.9 \%(0.5-2.5 \%)$ and a transferrin saturation of $4 \%$. There was no evidence of hemolysis as bilirubin was normal with normal lactate dehydrogenase of 354 IU/l (313-618 IU/l) and a normal haptoglobin level of $214 \mathrm{mg} / \mathrm{dl}(43-212 \mathrm{mg} / \mathrm{dl})$. She had a normal serum CEA level of $1.3 \mathrm{ng} / \mathrm{ml}(0-3.0 \mathrm{ng} / \mathrm{ml})$.

Subsequently she developed severe nausea with projectile vomiting. CT scan of abdomen and pelvis showed a small bowel lesion measuring about $4.1 \mathrm{~cm}$ in length with thickening of the wall and narrowing of the lumen of the involved segment (fig. 1). A long distended loop of small bowel was seen in the left abdomen and pelvis secondary to the obstructing lesion in the right lower quadrant. No focal 
hepatic lesions or any mesenteric or omental involvement were seen. Small bowel follow-through with barium showed an irregular filling defect in the jejunum suspicious for malignancy (fig. 2 ). She underwent resection of the jejunum which revealed a tumor of the small intestine with $4.5 \mathrm{~cm}$ in the greatest dimension (fig. 3 ). A separate tumor mass in the mesentery was seen. Pathology revealed moderately differentiated adenocarcinoma with involvement of the entire thickness of the intestinal wall. Six out of nine lymph nodes dissected were positive for metastatic carcinoma. Metastatic workup with CT scan of chest, abdomen and pelvis with oral and intravenous contrast revealed interval development of multiple hepatic lesions measuring about $1.5-2.0 \mathrm{~cm}$ with partial small bowel resection and without any evidence of bowel obstruction. Genetic studies of the specimen revealed that it had $K$ ras wild type mutation. She was started on palliative chemotherapy with a modified FOLFOX-6 regimen comprising leucovorin, oxaliplatin and 5-flurouracil for a total of 12 cycles. She tolerated chemotherapy well except for grade 1 oxaliplatin-induced peripheral neuropathy. She had a stable disease for over 6 months. In view of her K-ras wild type, she was stated on maintenance panitumumab and irinotecan and was doing well until the last follow-up.

\section{Discussion}

Here, we report a case of SBA with unexplained iron deficiency anemia despite extensive investigations for a year. The mass remained undetectable until the patient had a small bowel obstruction with metastatic disease involving the liver. A search of the US National Cancer Institute database has found $41.5 \%$ of cases of small bowel carcinoma which are similar in presentation and are diagnosed late as stage IV disease [8].

Several studies have reported that $6-12 \%$ of patients with occult gastrointestinal bleeding are diagnosed with small bowel tumors $[4,9]$. These patients are usually asymptomatic and their symptoms constitute nonspecific abdominal discomfort and abdominal pain. Later, these patients develop small bowel obstruction, which is somewhat similar to the presentation of our patient [10]. In a study conducted by Hong et al. [10] $(\mathrm{n}=22,41.5 \%)$ patients diagnosed as stage IV SBA had a median survival of 4 months. No statistically significant difference was noted in terms of histological grade, site of primary tumor and tumor markers. One of the major limiting factors for poor prognosis in such cases is emergent laparotomy performed by a non-oncologic surgeon which makes optimal radical resection generally impossible. Studies have shown that suboptimal resection is associated with inaccurate pathological staging as well as with a poorer patient outcome [10]. Unfortunately most chemotherapy regimen achieve a low response rate with a minimal survival benefit.

Several studies have explored the role of palliative chemotherapy in stage IV SBA. Hong et al. [10] have shown statistically significant survival in patients who received palliative chemotherapy ( 8 vs. 3 months) compared to those who did not receive chemotherapy $(\mathrm{p}=0.025)$. Gibson et al. [11] have shown a response rate of $18.4 \%$ and a median overall survival of 8 months in a series of 39 patients. These patients received 5-FU, adriamycin and mitomycin-C prospectively. More recently, a British study in 1998 by Crawley et al. [12] reported 8 patients with advanced SBA treated with an infusion 5 -FU-based regimen. They found a response rate of $37.5 \%$ and a median survival of 13 months. Fishman et al. [13] have shown a $30-40 \%$ response rate in patients who received gemcitabine and irinotecan-based regimen.

From the limited clinical reports, the regimen of 5-FU with platinum compounds has been acknowledged as the first-line treatment for palliative chemotherapy in metastatic SBA [14]. Irinotecan has a good response in advanced SBA as second-line agent. 
We noticed a response to chemotherapy for 6 months with FU-based regimen and a stable disease for another 6 months on second-line panitumumab (in view of $K$-ras wild type) and irinotecan and the patient is currently receiving the above regimen.

\section{Conclusion}

SBA remains a diagnostic challenge. Physician's suspicion and awareness is crucial in making appropriate diagnostic investigations for earlier detection of SBA. This increases the chance of resection and hence survival. This is especially true in cases of unexplained iron deficiency anemia - an uncommon presentation - and in patients with abdominal pain of unknown cause. Moreover, patients with delayed diagnosis often have poorer prognosis. In this case, we have noticed a modest response to palliative chemotherapy with modified FU-based regimen (5-FU, folinic acid and oxaliplatin). We believe that further studies are needed to evaluate the possible application of newer investigative agents and treatment strategies to obtain a better outcome.

\section{Acknowledgements}

None.

\section{Disclosure Statement}

None. 


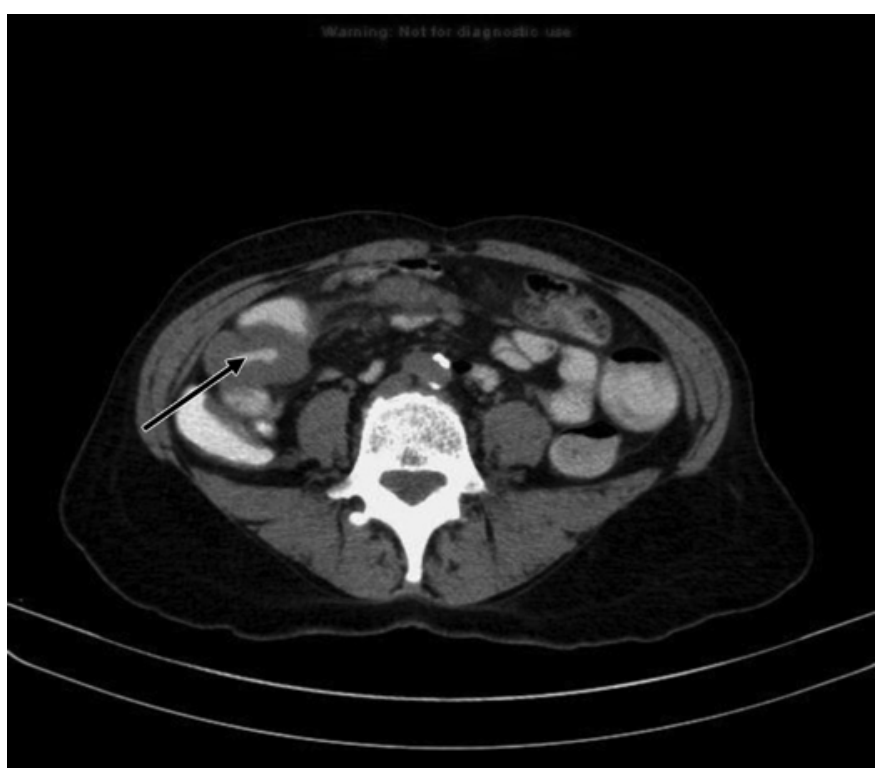

Fig. 1. CT Scan of abdomen with oral and IV contrast showing thickening of wall of the small intestine.

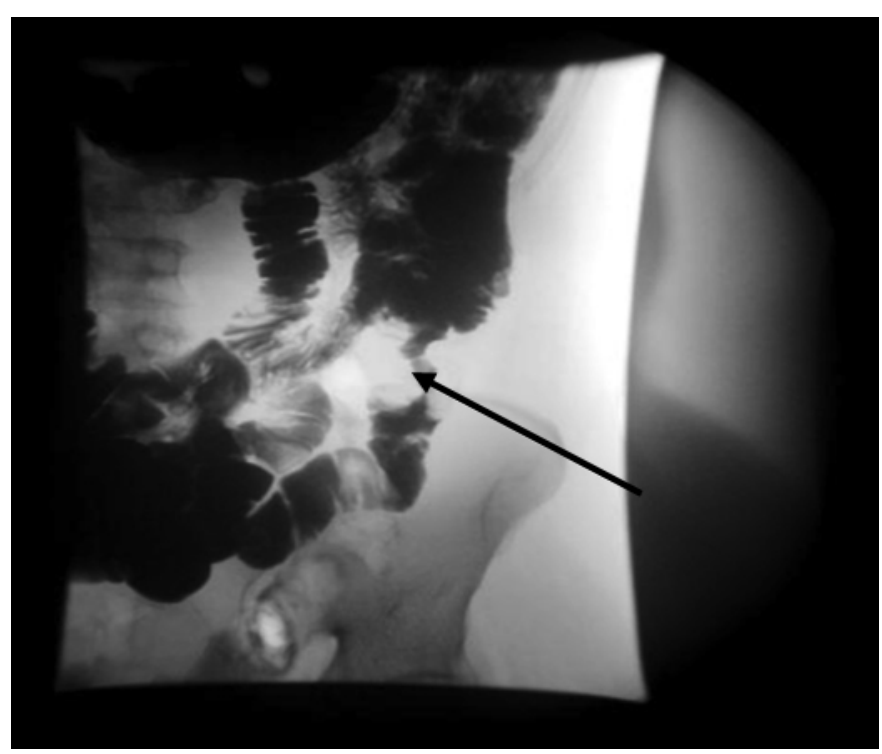

Fig. 2. Small bowel follow-through with barium showing irregular filling defect in jejunum. 


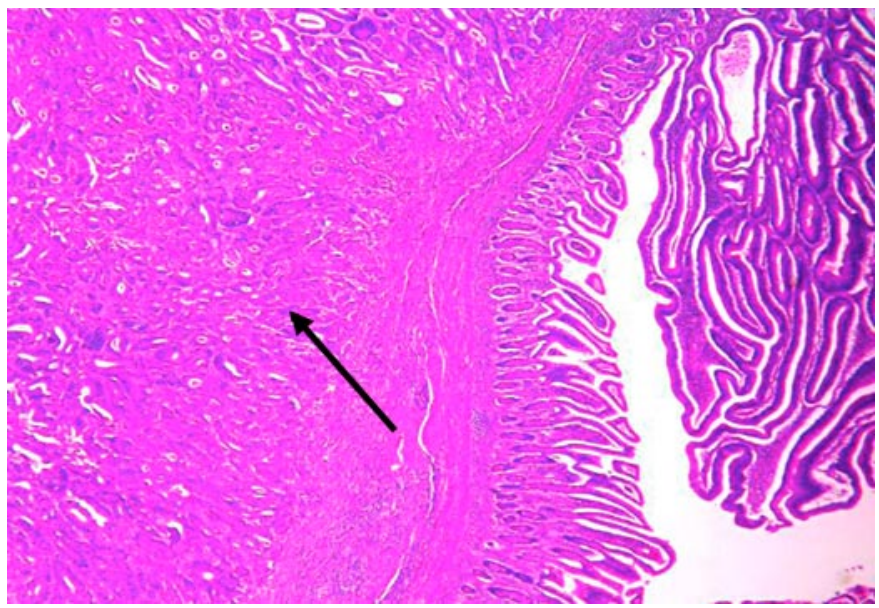

Fig. 3. Hematoxylin-eosin stain at $100 \times$ showing infiltrative small bowel adenocarcinoma with adjacent villous adenoma.

\section{References}

1 Ouriel K, Adams JT: Adenocarcinoma of the small intestine. Am J Surg Jan 1984;147:66-71.

2 Lewis BS: Enteroscopy; in Gourtsoyiannis N, Nolan D (eds): Imaging of Small Intestinal Tumors. New York, Elsevier, 1997, pp 375-390.

3 Haselkorn T, Whittemore AS, Lilienfeld DE: Incidence of small bowel cancer in the United States and worldwide: geographic, temporal, and racial differences. Cancer Causes Control 2005;16:781-787.

4 Cobrin GM, Pittman RH, Lewis BS: Increased diagnostic yield of small bowel tumors with capsule endoscopy. Cancer 2006;107:22-27.

5 Agrawal S, McCarron EC, Gibbs JF, et al: Surgical management and outcome in primary adenocarcinoma of the small bowel. Ann Surg Oncol 2007;14:2263-2269.

6 Dabaja BS, Suki D, Pro B, et al: Adenocarcinoma of the small bowel: presentation, prognostic factors, and outcome of 217 patients. Cancer 2004;101:518-526.

7 Maglinte DD, O'Connor K, Bessette J, et al: The role of the physician in the late diagnosis of primary malignant tumors of the small intestine. Am J Gastroenterol 1991;86:304-308.

-8 Howe JR, Karnell LH, Menck HR, et al: The American College of Surgeons Commission on Cancer and the American Cancer Society. Adenocarcinoma of the small bowel: review of the National Cancer Data Base, 19851995. Cancer 1999;86:2693-2706.

9 Heine GD, Hadithi M, Groenen MJ, et al: Double-balloon enteroscopy: indications, diagnostic yield, and complications in a series of 275 patients with suspected small-bowel disease. Endoscopy 2006;38:42-48.

10 Hong SH, Koh YH, Rho SY, et al: Primary adenocarcinoma of the small intestine: presentation, prognostic factors and clinical outcome. Jpn J Clin Oncol 2009;39:54.

11 Gibson MK, Holcroft CA, Kvols LK, et al: Phase II study of 5-fluorouracil, doxorubicin, and mitomycin C for metastatic small bowel adenocarcinoma. Oncologist 2005;10:132-137.

12 Crawley C, Ross P, Norman A, et al: The Royal Marsden experience of a small bowel adenocarcinoma treated with protracted venous infusion 5-fluorouracil. Br J Cancer 1998;78:508-510.

13 Fishman PN, Pond GR, Moore MJ, et al: Natural history and chemotherapy effectiveness for advanced adenocarcinoma of the small bowel: a retrospective review of 113 cases. Am J Clin Oncol 2006;29:225-231.

14 Locher C, Malka D, Boige V, et al: Combination chemotherapy in advanced small bowel adenocarcinoma. Oncology 2005;69:290-294. 